\title{
What is open?
}

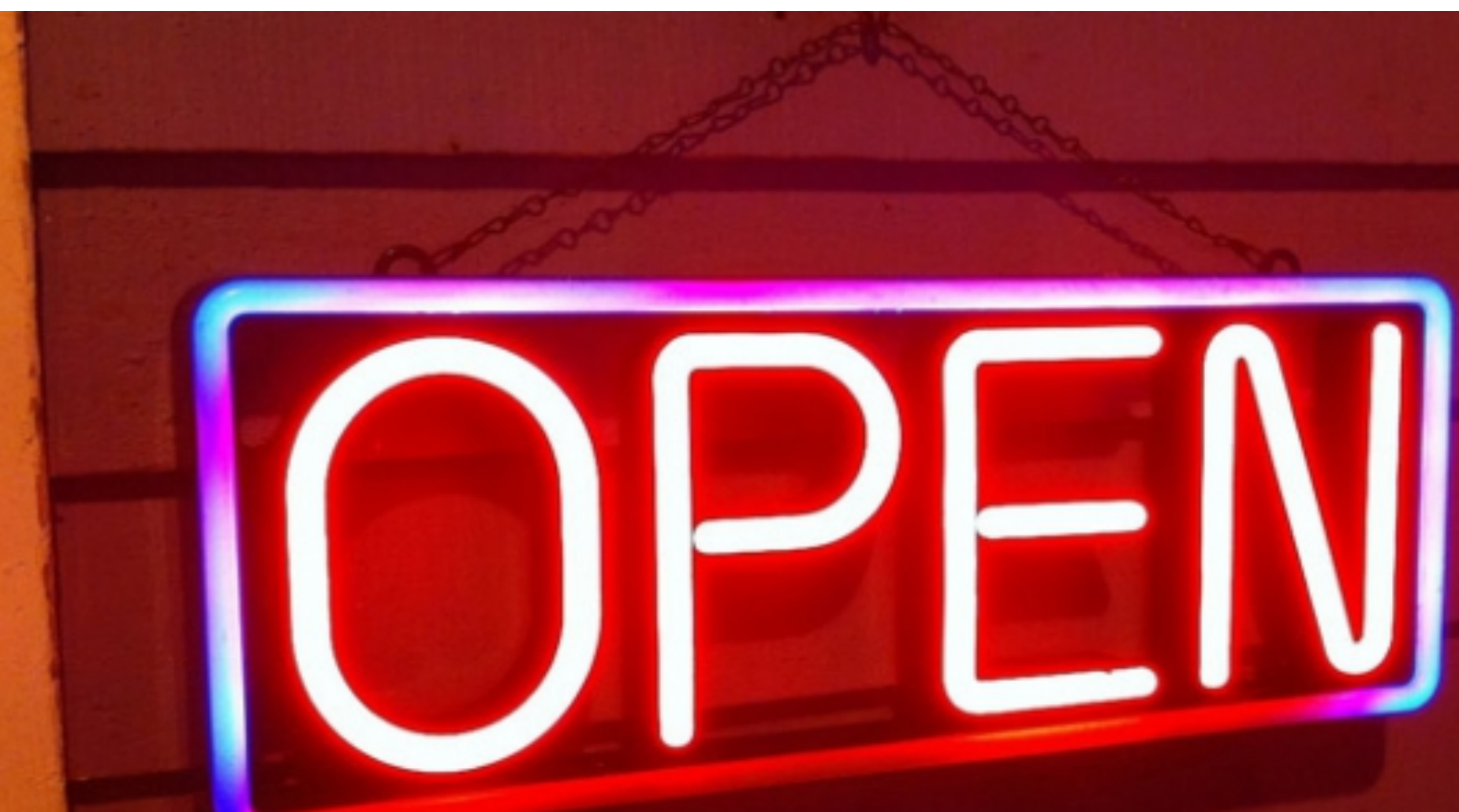




\section{Context}

- Openness can contribute to better research. areater impact, and value for money

More (and more

effective) scrutiny

- Available to more $y$ outputs and research
users

Easier to attempt

replication

- Research products

as inputs to new

research

- Openness is a spectrum

- Open implementation depends on numerous factors, including disciplinary values, cultures and practices, types of outputs, goals and practical considerations 
The open spectrum 


\section{Four dimension of openness}

a 1. Discoverable

国 2. Accessible

3. Reusable

目 4. Transparent 


\section{Discoverable}

- Good metadata available on the public Internet (including use of standards)

- Linked

- Persistent and unique identifiers

- Clear rights statements

(some level of discoverability is part of the baseline for openness) 


\section{Accessible}

- Free to all users at the point of use, and in perpetuity

baseline

- Readable by all regardless of disability

- Downloadable

- Machine readable

- It is more accessible, if it is open sooner 


\section{Reusable}

- Fewest restrictions on reuse, dissemination and modification 


\section{Transparent}

- Peer-review

- Impact metrics

- Transparency in the research process (e.g. TOP Guidelines)

- $\quad$ Citation standards

- Data transparency

- $\quad$ Analytical methods/code

- Research materials

- Design and analysis

- Pre-registration

- Replication

- Author Transparency - author names, roles, funding, institutional affiliations, other disclosures of potential conflict of interest 


\section{Next steps}

- Draft self-assessment framework

- Open for comments/feedback

- OSI branded publication? 
The 4-dimensional hypercube of openness

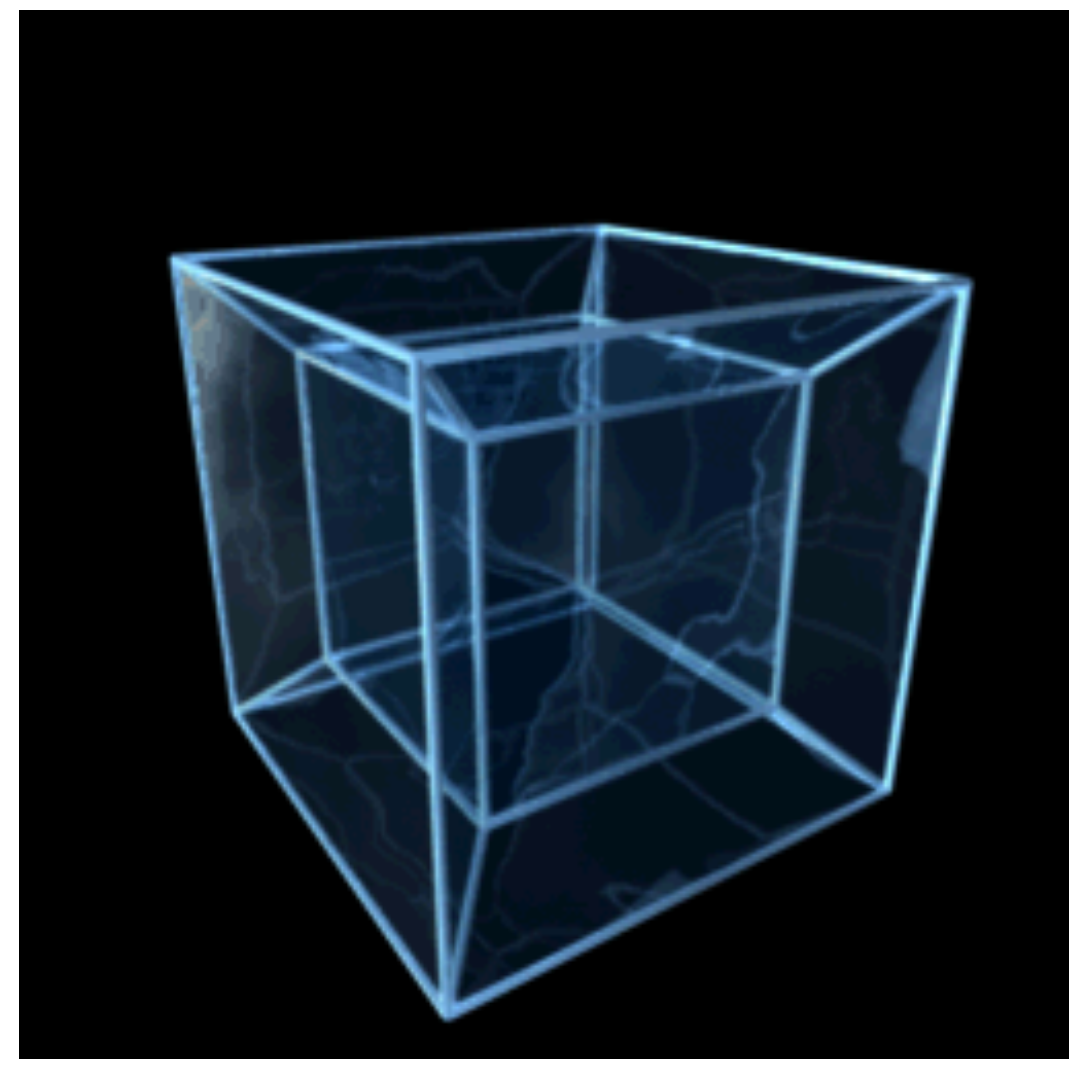

